\title{
QRS Slopes for Potassium and Calcium Monitoring in End-Stage Renal Disease Patients
}

\author{
Hassaan A Bukhari ${ }^{1,2,3}$, Pablo Laguna ${ }^{1,4}$, Mark Potse ${ }^{2,3}$, Carlos Sánchez $^{1,4}$, Esther Pueyo ${ }^{1,4}$ \\ ${ }^{1}$ I3A, University of Zaragoza, IIS Aragón, Zaragoza, Spain \\ ${ }^{2}$ Univ. Bordeaux, IMB, UMR 5251, Talence, France \\ ${ }^{3}$ Carmen Team, Inria Bordeaux - Sud-Ouest \\ ${ }^{4}$ CIBER en Bioingeniería, Biomateriales y Nanomedicina, Spain
}

\begin{abstract}
Non-invasive estimation of serum potassium, $\left[\mathrm{K}^{+}\right]$, and calcium, $\left[\mathrm{Ca}^{2+}\right]$, are of major importance to prevent ventricular arrhythmias and sudden cardiac death, but current ambulatory estimation methods, mostly based on $T$ wave properties, are limited. We present an analysis based on QRS slopes to detect and quantify electrolyte abnormalities in end-stage renal disease (ESRD) patients.

We applied principal component analysis to 12-lead electrocardiograms (ECGs) of 29 ESRD patients undergoing hemodialysis (HD). In the first principal component, we analyzed two-minute segments at the end of each HD hour and computed a mean warped $Q R S$ complex (MWQRS) representing an optimal average of QRS complexes. We calculated the upward ( $\left.I_{\mathrm{US}}\right)$ and downward $\left(I_{\mathrm{DS}}\right)$ slope of the MWQRS and we quantified the relative slope changes with respect to the end of the $H D$ session ( $\Delta I_{\mathrm{US}}$ and $\Delta I_{\mathrm{DS}}$ ).

$\Delta I_{\mathrm{US}}$ and $\Delta I_{\mathrm{DS}}$ significantly increased and decreased, respectively, during $H D$ in association with decreasing $\left[\mathrm{K}^{+}\right]$and increasing $\left[\mathrm{Ca}^{2+}\right]$. The median correlation coefficients of $\Delta I_{\mathrm{US}}$ and $\Delta I_{\mathrm{DS}}$ with the change in $\left[\mathrm{K}^{+}\right]$were -0.84 and 0.88 , respectively. Corresponding correlation coefficients with the change in $\left[\mathrm{Ca}^{2+}\right]$ were 0.78 and -0.91 . The quantified QRS slope changes were related to both amplitude and duration changes in the QRS complex during HD.

Changes in QRS slopes are strongly related to variations in $\left[\mathrm{K}^{+}\right]$and $\left[\mathrm{Ca}^{2+}\right]$ during $H D$ and could be used to monitor electrolyte concentrations in ESRD patients.
\end{abstract}

\section{Introduction}

Chronic kidney disease (CKD) affects around 10\% of humanity, with high economic cost, increased mortality risk and decreased quality of life [1]. At the final stage of CKD, end-stage renal disease (ESRD) patients present decreased ability to maintain potassium and calcium homeostasis. Serum potassium $\left(\left[\mathrm{K}^{+}\right]\right)$and calcium $\left(\left[\mathrm{Ca}^{2+}\right]\right)$ levels outside normal ranges, in the form of hypoor hyperkalemia and hypo- or hypercalcemia, increase the risk for life-threatening arrhythmias and sudden cardiac death $[2,3]$.

Electrocardiogram (ECG) depolarization and repolarization are known to be affected by variations in $\left[\mathrm{K}^{+}\right]$and $\left[\mathrm{Ca}^{2+}\right][2-4]$, which has been used to derive markers for continuous monitoring of $\left[\mathrm{K}^{+}\right]$and $\left[\mathrm{Ca}^{2+}\right]$. In a recently published study [5], several $\mathrm{T}$ wave indices are featured as candidates for $\left[\mathrm{K}^{+}\right]$and $\left[\mathrm{Ca}^{2+}\right]$ monitoring. However, the majority of these features do not account for changes in the whole ECG morphology induced by $\left[\mathrm{K}^{+}\right]$and $\left[\mathrm{Ca}^{2+}\right]$ variations but most commonly refer to a specific time point or small portion of the $\mathrm{T}$ wave.

In previous studies [6-8], we used time-warping techniques to characterize changes in the shape of the $\mathrm{T}$ wave at varying $\left[\mathrm{K}^{+}\right]$in patients and simulated ECGs. Also, we investigated changes in the nonlinear dynamics of the $\mathrm{T}$ wave at varying $\left[\mathrm{K}^{+}\right]$[9]. A strong relationship was found between investigated linear and nonlinear features of the $\mathrm{T}$ wave and $\left[\mathrm{K}^{+}\right][8,10]$. To further improve prediction of electrolyte levels, ECG markers characterizing the $\mathrm{T}$ wave could be combined with markers of the QRS complex, which is also known to be affected by varying $\left[\mathrm{K}^{+}\right]$and $\left[\mathrm{Ca}^{2+}\right][2-4]$.

In this study, we characterized the morphology of the QRS complex by quantifying its upward ( $\left.I_{\mathrm{US}}\right)$ and downward $\left(I_{\mathrm{DS}}\right)$ slopes during hemodialysis (HD) in ESRD patients. We investigated how changes in $I_{\mathrm{US}}$ and $I_{\mathrm{DS}}$ were related to $\left[\mathrm{K}^{+}\right],\left[\mathrm{Ca}^{2+}\right]$ and heart rate $(\mathrm{HR})$ variations. 


\section{Methods}

\subsection{Study population}

The study population comprised 29 ESRD patients from Hospital Clínico Universitario de Zaragoza (HCUZ), with 48-hour 12-lead ECGs. ECG acquisition started 5 minutes before the onset of HD and lasted 48 hours, as shown in Figure 1 [6-8]. Concurrently, six blood samples were taken at the HD onset, at every hour during the HD session, at the HD end (minute 215 or 245 , depending on the patient) and after 48 hours, immediately before the next HD session (Figure $1, h_{0}$ to $h_{48}$ ). $\left[\mathrm{K}^{+}\right]$and $\left[\mathrm{Ca}^{2+}\right]$ as well as $\mathrm{HR}$ were measured at those time points. All patients provided signed informed consent. The ethical committee approved the study protocol (CEICA ref. PI18/003).

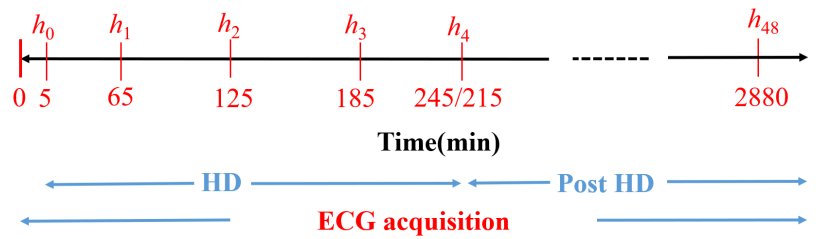

Figure 1. Diagram of the study protocol. $h_{0}$ to $h_{48}$ are the time points (in minutes) for blood sample extraction.

\subsection{Preprocessing of ECG signals}

A wavelet-based algorithm was used for single-lead delineation of the ECG [11]. Baseline wander, muscle and electric noise was attenuated by proper filtering [12]. Spatial principal components (PCs) were obtained by computing the inter-lead auto-correlation matrix of QRS complexes in a stable ECG segment at the end of the HD session. The first PC was used to enhance the QRS complex energy and allow more precise delineation [13]. The locations of the QRS onset, peak and end were identified.

Mean warped QRS complexes (MWQRS) were calculated from aligned QRS complexes at two-minutes windows centered at each of the analyzed time points when blood samples were extracted, representing optimal QRS averages [13]. Each MWQRS, calculated at $h_{0}, h_{1}, h_{2}, h_{3}$ and $h_{4}$, is denoted by $x_{\mathrm{QRS}}(n)$.

\subsection{Measurement of QRS Slopes}

From each MWQRS, the upward slope, $I_{\mathrm{US}}$, and the downward slope, $I_{\mathrm{DS}}$, were computed $[14,15] . I_{\mathrm{US}}$ and $I_{\mathrm{DS}}$ were defined as the maximum and minimum value of the MWQRS complex derivative denoted by $x_{\mathrm{QRS}}^{\prime}(n)$,

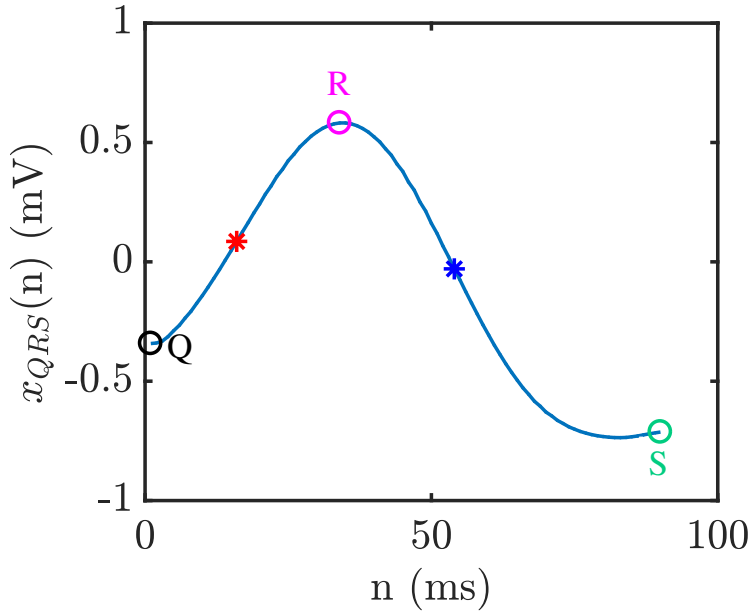

Figure 2. MWQRS from a patient's ECG at the start of HD $\left(h_{0}\right)$. Locations of Q (onset of MWQRS), R (peak of MWQRS) and S (end of MWQRS) waves are marked, as well as the locations of maximum and minimum QRS complex derivative (red and blue asterisks, respectively).

respectively:

$$
\begin{aligned}
& I_{\mathrm{US}}=\max _{n}\left\{x_{\mathrm{QRS}}^{\prime}(n)\right\}, \quad n \in\{Q+1, \ldots, R-1\}, \\
& I_{\mathrm{DS}}=\min _{n}\left\{x_{\mathrm{QRS}}^{\prime}(n)\right\}, \quad n \in\{R+1, \ldots, S-1\},
\end{aligned}
$$

with

$$
x_{\mathrm{QRS}}^{\prime}(n)=0.5 \cdot\left(x_{\mathrm{QRS}}(n+1)-x_{\mathrm{QRS}}(n-1)\right),
$$

and $Q, R$ and $S$ being the sample locations of the onset, peak and end of the MWQRS, respectively (see Figure 2). For time samples $n$ at the limits of the QRS upward and downward intervals, the derivative computation in equation (3) require values outside the interval. In such cases, those values are replaced with the ones at the interval limit. Both slopes are expressed in $\mathrm{mV} / \mathrm{ms}$.

From the values of $I_{\mathrm{US}}$ and $I_{\mathrm{DS}}$ along HD, we quantified the change in QRS slopes with respect to the end of the HD session $\left(h_{4}\right)$ and we denoted them as $\Delta I_{\mathrm{US}}$ and $\Delta I_{\mathrm{DS}}$.

The Pearson correlation coefficient $\rho$ and the coefficient of determination $R^{2}$ between $\Delta I_{\mathrm{US}}$ ( $\Delta I_{\mathrm{DS}}$, respectively) and the changes in $\left[\mathrm{K}^{+}\right],\left[\mathrm{Ca}^{2+}\right]$ and $\mathrm{RR}$ were computed to assess the strength of electrolyte and heart rate variations on QRS slopes during HD.

\section{Results and Discussion}

Fig. 3 shows the relationship between $\Delta I_{\mathrm{US}}\left(\Delta I_{\mathrm{DS}}\right.$, respectively) and $\Delta\left[\mathrm{K}^{+}\right]$for the 29 patients (panels a-b). $\Delta I_{\mathrm{US}}$ and $\Delta I_{\mathrm{DS}}$ took the smallest and largest values at the beginning of the HD session, corresponding to 

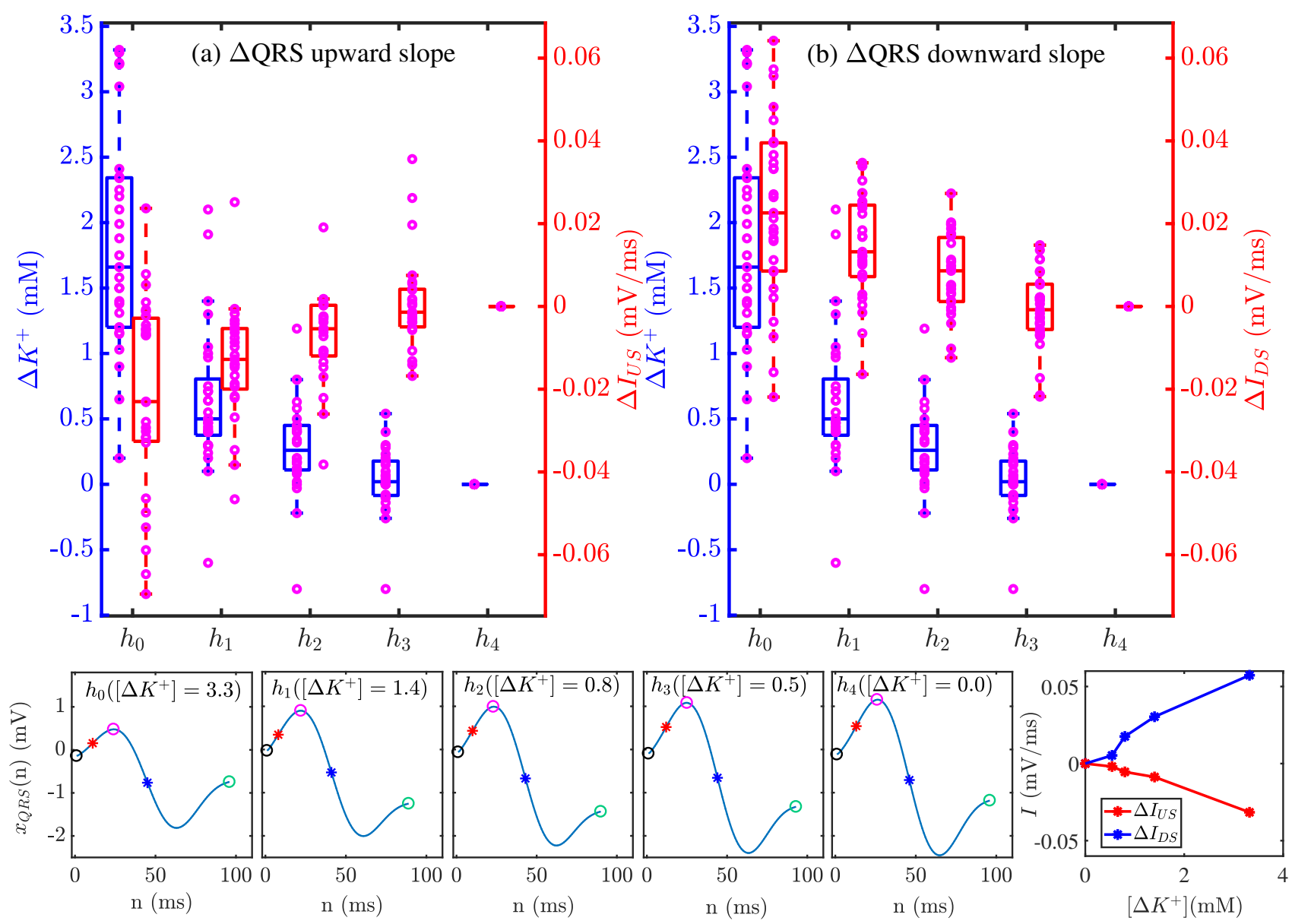

Figure 3. Panels a-b: Relationships of $\Delta I_{\mathrm{US}}$ (red) and $\Delta I_{\mathrm{DS}}$ (red) with $\Delta\left[\mathrm{K}^{+}\right]$(blue) from MWQRS of 29 ESRD patients. The central line indicates the median, whereas top and bottom edges show the $25 t h$ and $75 t h$ percentiles. Each purple dot corresponds to an individual patient. Bottom panels show the MWQRS complexes and QRS slopes variations during HD with $\Delta\left[\mathrm{K}^{+}\right]$for a patient. Location of $\mathrm{Q}, \mathrm{R}$ and $\mathrm{S}$ waves were marked by black, purple and green circles, respectively. Red and blue asterisks show the locations associated with $I_{\mathrm{US}}$ and $I_{\mathrm{DS}}$ in the QRS complex, respectively. $\Delta$ denotes change with respect to the end of HD session $\left(h_{4}\right)$.

the highest $\left[\mathrm{K}^{+}\right]$values, respectively. In the bottom panels of Fig. 3, significant changes in the morphology of the QRS complex can be observed during HD. Both QRS markers significantly varied with decreasing $\left[\mathrm{K}^{+}\right]$ during HD, in all the ESRD patients. As shown in the figure, QRS slope changes in ESRD patients were related to both amplitude and duration of the QRS complex.

Correlation analysis was performed to assess the relationship between $\Delta\left[\mathrm{K}^{+}\right], \Delta\left[\mathrm{Ca}^{2+}\right]$ or $\Delta \mathrm{RR}$ variations (representing changes in $\left[\mathrm{K}^{+}\right],\left[\mathrm{Ca}^{2+}\right]$ and $\mathrm{RR}$ with respect to $\mathrm{HD}$ end) and the corresponding changes in $\Delta I_{\mathrm{US}}$ and $\Delta I_{\mathrm{DS}}$. Figure 4 shows the strong linear Pearson correlation of $\Delta I_{\mathrm{US}}$ and $\Delta I_{\mathrm{DS}}$ with $\Delta\left[\mathrm{K}^{+}\right]$(median value of -0.84 and 0.88 ) and with $\Delta\left[\mathrm{Ca}^{2+}\right]$ (median value of 0.78 and -0.91 ). However, a poor association was found between both markers and $\Delta$ RR because the $R R$ interval varies much less than $\left[\mathrm{K}^{+}\right]$and $\left[\mathrm{Ca}^{2+}\right]$ during $\mathrm{HD}$ in the analyzed ESRD patients. The $R^{2}$ values between $\Delta I_{\mathrm{US}}$ and $\Delta I_{\mathrm{DS}}$ with $\Delta\left[\mathrm{K}^{+}\right]$were 0.71 and 0.79 , and with $\Delta\left[\mathrm{Ca}^{2+}\right]$ these were 0.66 and 0.85 . As an observation from Figure 4, a wide range of patterns was obtained for the investigated relationships. Computational modeling and simulation of human ventricular electrophysiology could help to gain insight into the sources of such high inter-patient variability.

\section{Conclusions}

QRS slopes-based markers present remarkable changes with varying $\left[\mathrm{K}^{+}\right]$and $\left[\mathrm{Ca}^{2+}\right]$ in ESRD patients. These results emphasize the importance of characterizing ECG depolarization, in addition to ECG repolarization, 


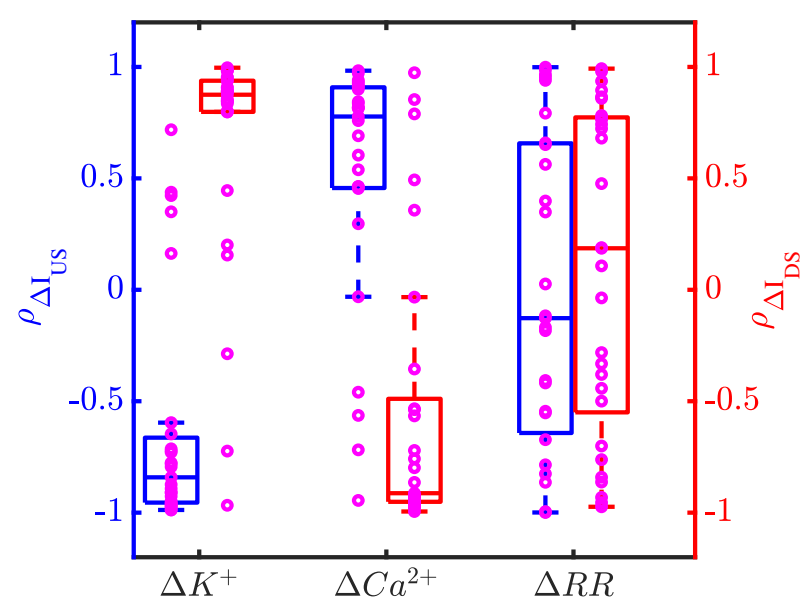

Figure 4. Median (25th/75th percentiles) of $\rho$ between $\Delta I_{\mathrm{US}}$ (blue) or $\Delta I_{\mathrm{DS}}$ (red) and $\Delta\left[\mathrm{K}^{+}\right], \Delta\left[\mathrm{Ca}^{2+}\right]$ and $\Delta$ RR. $\Delta$ denotes change with respect to the end of HD session.

to monitor hypo- or hyperkalemia and hypo- or hypercalcemia, which could be used to predict arrhythmic events in these patients.

\section{Acknowledgements}

This work was supported by projects ERC-StG 638284 (ERC), PID2019-105674RB-I00 and PID2019-104881RB-I00 (Ministerio de Ciencia e Innovación), Marie Skłodowska-Curie grant 764738 (European Commission) and European Social Fund (EU) and Aragón Government through BSICoS group T39_20R and project LMP124-18. Computations were performed by ICTS NANBIOSIS (HPC Unit, Univ. Zaragoza).

\section{References}

[1] Hill NR, et al. Global prevalence of chronic kidney disease - A systematic review and meta-analysis. PloS One 2016; 11(7):e0158765.

[2] Weiss JN, et al. Electrophysiology of hypokalemia and hyperkalemia. Circulation Arrhythmia and Electrophysiology 2017;10(3).

[3] Noordam R, et al. Effects of calcium, magnesium, and potassium concentrations on ventricular repolarization in unselected individuals. Journal of the American College of Cardiology June 2019;73(24):3118-3131.

[4] El-Sherif $\mathrm{N}$, et al. Electrolyte disorders and arrhythmogenesis. Cardiology Journal 2011; 18(3):233-245

[5] Pilia N, et al. Quantification and classification of potassium and calcium disorders with the electrocardiogram: What do clinical studies, modeling, and reconstruction tell us? APL Bioengineering October 2020;4(4):041501.

[6] Palmieri F, et al. Monitoring blood potassium concentration in hemodialysis patients by quantifying T-wave morphology dynamics. Scientific Reports February 2021;11(1):3883.

[7] Bukhari HA, et al. Transmural ventricular heterogeneities play a major role in determining $\mathrm{T}$-wave morphology at different extracellular potassium levels. In 2019 Computing in Cardiology. 2019; 1-4.

[8] Bukhari HA, et al. Characterization of T wave amplitude, duration and morphology changes during hemodialysis: Relationship with serum electrolyte levels and heart rate. IEEE transactions on bio medical engineering August 2021; 68(8):2467-2478.

[9] Srinivasan S, et al. Analysis of T wave nonlinear dynamics for serum potassium monitoring in end-stage renal disease patients. In 2020 Computing in Cardiology. 2020; 1-4.

[10] Bukhari HA, et al. Estimation of potassium levels in hemodialysis patients by $\mathrm{T}$ wave nonlinear dynamics and morphology markers. IEEE Transactions on Biomedical Engineering 2021, Under Review;

[11] Martínez JP, et al. A wavelet-based ECG delineator: evaluation on standard databases. IEEE Transactions on Biomedical Engineering April 2004;51(4):570-581.

[12] Sörnmo L, Laguna P. Electrocardiogram (ECG) signal processing. In Wiley Encyclopedia of Biomedical Engineering. American Cancer Society. ISBN 978-0-471-74036-0, 2006;

[13] Bukhari HA, et al. Ambulatory monitoring of serum potassium and calcium levels in end-stage renal disease patients by combined ECG depolarization and repolarization morphology analysis. IEEE Transactions on Biomedical Engineering 2021, to be submitted;

[14] Pueyo E, et al. QRS slopes for detection and characterization of myocardial ischemia. IEEE transactions on bio medical engineering February 2008;55(2):468-477.

[15] Ringborn M, et al. Evaluation of depolarization changes during acute myocardial ischemia by analysis of QRS slopes. Journal of Electrocardiology August 2011; 44(4):416-424.

Address for correspondence:

Hassaan A. Bukhari; Institute of Engineering Research of Aragón, Universidad de Zaragoza (Spain).

hassaanahmed01@unizar.es 